\title{
PLASMA,
}

GASES

\section{Hierarchical Pattern of Superdiffusion}

\author{
A. I. Olemskor \\ Sumy State University, Sumy, 244007 Ukraine \\ e-mail: olemskoi@ssu.sumy.ua \\ Received February 23, 2000
}

\begin{abstract}
Under the assumption of slow evolution of the cluster structure of walker positions, the kinetic equation is derived, according to which the Levy law is fulfilled on the mesoscopic time-scale, while the system tends to the generalized Tsallis distribution on the macroscopic time-scale. The asymptotics are found for the time dependences of the hierarchy characteristic scale and the probability distribution over the hierarchical levels. (C) 2000 MAIK "Nauka/Interperiodica".
\end{abstract}

PACS numbers: 5.20.Db, 5.40.+j, 64.40.Ak

As known, the stochastic transfer processes are the generalization of the diffusion process [1]. They are characterized by the transition from the ordinary square-root law to the relation

$$
\left\langle\mathbf{r}^{2}\right\rangle \propto t^{2 / z},
$$

with the dynamic exponent $z \neq 2$ ( $\mathbf{r}$ is the coordinate of a walking particle and $t$ is time). For the subdiffusion in the presence of traps, the jump mean waiting time diverges, $\langle t\rangle=\infty$, as a result of which the jumps occur discretely in space and the transfer process is decelerated $(z>2)$. It accelerates $(z<2)$ in the presence of Levy flights when the particle executes jumps of arbitrary length with the divergent mean square displacement $\left\langle\mathbf{x}^{2}\right\rangle=\infty$ at discrete time instants.

A remarkable feature of the superdiffusion process is that the successive positions of the walking particle form cluster structure representing a fractal set with the dimension of exponent $z$ [2]. Since the fractal is formed by the hierarchical construction, one can assume that the behavior of the stochastic system is determined not only by the particle displacement in the real space but also by a much slower evolution of the clusters of its successive positions. This evolution is known to amount to the diffusion over the sites of a hierarchical tree in the ultrametric space.

This study is devoted to the description of the superdiffusion as a random walk in the real and ultrametric spaces. The generalized Fokker-Planck equation will be used to show that the particle positions conform to the Levy distribution on the mesoscopic time-scale, where the cluster structure does not undergo noticeable changes. Similar to the slow relaxation of spin glasses, it should be expected that on the macroscopic timescale the cluster structure would undergo noticeable changes on the way to the stationary distribution of particle positions. Such a behavior results in the Tsallis form of asymptotic distribution, which, in turn, corre- sponds to the highest level of the particle-position cluster distribution [3].

Let us first present the information necessary for the description of the superdiffusion in the case when the cluster structure is ignored [4]. The initial kinetic equation has the form

$$
\begin{gathered}
P\left(\mathbf{r}, t+\tau_{0}\right)-P(\mathbf{r}, t) \\
=\int\left[f\left(\mathbf{r}, \mathbf{r}^{\prime}\right) P\left(\mathbf{r}^{\prime}, t\right)-f\left(\mathbf{r}^{\prime}, \mathbf{r}\right) P(\mathbf{r}, t)\right] d \mathbf{r}^{\prime},
\end{gathered}
$$

where $\tau_{0}$ is the jump time and $f\left(\mathbf{r}, \mathbf{r}^{\prime}\right)$ is the probability of transition from point $\mathbf{r}^{\prime}$ to point $\mathbf{r}$. At $t \gg \tau_{0}$, the lefthand side of Eq. (2) amounts to the time derivative $\dot{P}(\mathbf{r}, t)$, and it is convenient, under the assumption of spatial homogeneity, to pass over to the integration with respect to the displacement $\mathbf{x} \equiv \mathbf{r}-\mathbf{r}^{\prime}$ in the right-hand side. Then, using the detailed balancing principle $f(\mathbf{x})=$ $f(-\mathbf{x})$, one can recast the kinetic equation in the form

$$
\tau_{0} \dot{P}(\mathbf{r}, t)=\int[P(\mathbf{r}+\mathbf{x}, t)-P(\mathbf{r}, t)] f(\mathbf{x}) d \mathbf{x} .
$$

Taking into account the normalization condition $\int_{-\infty}^{\infty} f(\mathbf{x}) d \mathbf{x}=1$ for the space Fourier transforms, one obtains $\tau_{0} \dot{P}_{\mathbf{k}}(t)=-\left(1-f_{\mathbf{k}}\right) P_{\mathbf{k}}(t)$, where $\mathbf{k} \longrightarrow 0$ is the wave vector multiplied by the real-space characteristic scale. In the superdiffusion processes, of crucial importance is the behavior of the transition probability at large distances, $f(\mathbf{x}) \sim|\mathbf{x}|^{-(d+z)}$, where $d$ is the space dimensionality. In the corresponding $\mathbf{k} \longrightarrow 0$ limit, the $1-f_{\mathbf{k}}$ factor is reduced to $D_{z}|\mathbf{k}|^{z}$, where $D_{z}$ is the effective diffusion coefficient whose explicit expression is determined by the $f(\mathbf{x})$ function (in particular, for $d=1$ it was found that $D_{z}=2^{-z} \Gamma(1-z / 2) / \Gamma(1+z / 2)$ where $\Gamma(\xi)$ is 

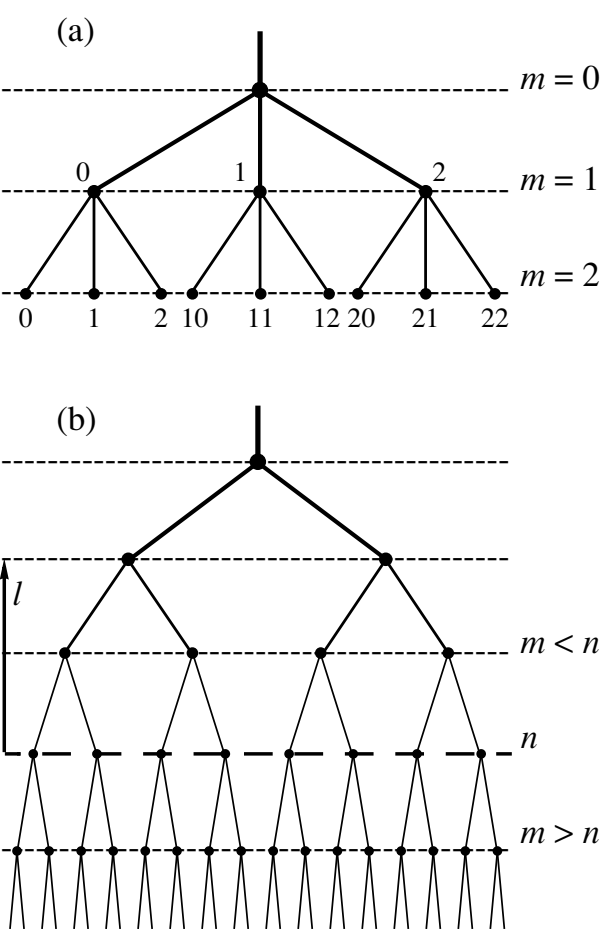

Fig. 1. Simplest hierarchical trees: (a) tree parametrization with the branching ratio $s=3$ and (b) bifurcation tree, $s=2$.

the gamma function [4]). As a result, one arrives at the Levy distribution

$$
P_{\mathbf{k}}(t)=P_{\mathbf{k}}(0) \exp \left(-t / \tau_{\mathbf{k}}\right), \quad \tau_{\mathbf{k}} \equiv D_{z}^{-1}|\mathbf{k}|^{-z} \tau_{0},
$$

characterized by the mesoscopic time $\tau_{\mathbf{k}} \gg \tau_{0}$. The corresponding kinetic equation in the real space contains fractional derivative of order $z$.

When the cluster structure is taken into account, the probability density $P_{\mathbf{u}}(\mathbf{r}, t)$ and the transition intensities $f_{\mathbf{u u}}\left(\mathbf{r}, \mathbf{r}^{\prime}\right)$ in kinetic equation (2) become dependent on the ultrametric coordinate $\mathbf{u}$ [5]. To reveal this dependence, let us consider a regular hierarchical tree characterized by a fixed branching ratio $s>1$ and the number $n \gg 1$ of hierarchy levels. In this case, the ultrametric coordinate $\mathbf{u}$ is an $n$-valued number in the $s$-digit number system: $\mathbf{u} \equiv u_{0} u_{1} \ldots u_{m} \ldots u_{n-1}, u_{m}=0,1, \ldots, s-1$ (an example is shown in Fig. 1a). Accordingly, the transition intensity is written as a power series

$$
f_{\mathbf{u u}^{\prime}}=\sum_{m=0}^{n} f\left(u_{m}-u_{m}^{\prime}\right) s^{n-m},
$$

where the first term $(m=0)$ corresponds to the highest hierarchy level governing the behavior of the whole system, while the last term $(m=n)$ corresponds to the lowest level representing the smallest clusters. By definition, the distance between the points $\mathbf{u}$ and $\mathbf{u}^{\prime}$ is $0 \leq$ $l \leq n$ if the conditions $u_{m}=u_{m}^{\prime}$ are fulfilled for $m=0,1$, $\ldots, n-(l+1)$ but $u_{m} \neq u_{m}^{\prime}$ for $m=n-1, n-l+1, \ldots$, $n$ [6]. For this reason, at a fixed distance $l$, the first $n-l$ terms of the above series are zero by definition, while the last $l$ terms contain the $s^{n-m}$ multiplier that in the continuous limit $s \gg 1$ is much smaller than the $s^{l}$ multiplier in the first of the remaining terms. As a result, the term corresponding to $m=n-l: f_{\text {uu' }} \sim s^{l}=s^{n-m}$, is the only leading term of the power series under consideration. Similarly, one can show that the probability density $P_{\mathbf{u}} \sim s^{n-l}=s^{m}$. Upon passing from the regular to the arbitrary tree [7], the branching ratio $s$ becomes the variable quantity and, according to the above estimates, the transition intensities $f_{\text {uu' }} \longrightarrow f(n-m)$ and the probability density $P_{\mathbf{k u}} \longrightarrow P_{\mathbf{k}}(m)$ take the form of Mellin transform:

$$
f(n-m) \equiv \int_{0}^{\infty} f(s) s^{n-m} d s, \quad P_{\mathbf{k}}(m) \equiv \int_{0}^{\infty} P_{\mathbf{k}}(s) s^{m} d s,
$$

where $f(s)$ and $P(s)$ are the respective weight functions.

The kinetic equation allowing for the cluster structure has the form

$$
\begin{gathered}
\tau_{\mathbf{k}} \dot{P}_{\mathbf{k}}(n, t)=\sum_{m>n} f(m-n) P_{\mathbf{k}}(n, t) \\
-\sum_{m<n} f(n-m) P_{\mathbf{k}}(m, t) .
\end{gathered}
$$

Here, the first term on the right-hand side allows for the hierarchical interrelation between the sites of the lower levels $m>n$ through a given $n$, while the subtracted term allows for the interrelation of a given level $n$ through the upper levels $m<n$ (see Fig. 1b). In deriving Eq. (5), the adiabatic approximation was used because the particle walks occur much rapidly than do the cluster structure changes on a macroscopic timescale $\tau$ (see below). Expanding $P_{\mathbf{k}}(m, t)$ in $n-m$ to the quadratic term, one obtains the following equation in the continuous limit $n \gg 1$ :

$$
\begin{aligned}
& \tau_{\mathbf{k}} \dot{P}_{\mathbf{k}}(n, t)=\frac{\partial}{\partial n}[\left.F(n) P_{\mathbf{k}}(n, t)-\frac{\partial}{\partial n} D(n) P_{\mathbf{k}}(n, t)\right] \\
&+\mathscr{D} P_{\mathbf{k}}(n, t)
\end{aligned}
$$

where the moments

$$
\begin{gathered}
F(n) \equiv \sum_{m<n}(n-m) f(n-m), \\
2 D(n) \equiv \sum_{m<n}(n-m)^{2} f(n-m)
\end{gathered}
$$

JETP LETTERS $\quad$ Vol. $71 \quad$ No. $7 \quad 2000$ 
and the quantity

$$
\mathscr{D} \equiv \sum_{m>n} f(m-n)-\sum_{m<n} f(n-m),
$$

specifying the difference between the rates of establishing hierarchical interrelation through the upper and lower levels are introduced.

The restrictions $m>n$ and $m<n$ are usually absent in the summation over the system states in Eq. (7), so that $\mathscr{D}=0$ [8]. However, it is beyond reason to formulate such a condition for the hierarchical systems, where the rate of establishing the interrelation essentially depends on the (levels upper or lower) mediating this process. Such is the case because the ultrametric space is inhomogeneous, as is evident from its geometric imaging (Fig. 1). For this reason, the following ansatz (the basic assumption) is adopted in this work:

$$
\mathscr{D} \equiv-\epsilon q P_{\mathbf{k}}^{q-1}(n, t) \frac{\partial}{\partial n},
$$

where $q$ and $\epsilon$ are the positive parameters. This ansatz is formally justified by the fact that the integral $\int \mathscr{D} P d n$ is reduced, within a factor of $-\epsilon(q-1)$ to the Jackson derivative with exponent $\alpha_{q}=(q-1) \ln P / \ln q$ [9]. As distinct from the ordinary derivative corresponding to the $q \longrightarrow 1$ limit, the Jackson derivative determines the rate of changing the $P(n)$ function not upon the argument displacement $d n \longrightarrow 0$ but upon its dilatation $q n$ and, therefore, provides the basis for an analysis of the self-similar systems. From the physical point of view, the fact that the difference $\mathscr{D}$ between the rates of establishing the hierarchical interrelation depends on the probability density $P_{\mathbf{k}}(n, t)$ implies the existence of a nonlinear feedback, which, as is seen from what follows, is responsible for the nonadditivity. Substitution of Eq. (8) in Eq. (6) results in the following kinetic equation of superdiffusion:

$$
\begin{gathered}
\left(\tau_{\mathbf{k}} \dot{P}_{\mathbf{k}}(n, t)=\frac{\partial}{\partial n}\right) \\
\times\left\{\left[F(n) P_{\mathbf{k}}(n, t)-\epsilon P_{\mathbf{k}}^{0}(n, t)\right]-\frac{\partial}{\partial n} D(n) P_{\mathbf{k}}(n, t)\right\} .
\end{gathered}
$$

Compared to the ordinary systems [8], the opposite signs of the diffusion and the linear-drift terms are noteworthy; this is caused by the choice of the opposite signs in initial Eq. (5). This sign difference is due to the fact that the autonomous hierarchical (e.g., bureaucratic) systems are not decomposed but spontaneously reproduced [7]. Note also that the nonlinearity of Eq. (9) does not allow one to use Mellin transform (4), as it was done for the Fourier transform of Eq. (2).

Turning to an analysis of Eq. (9), let us first consider the case $F(n)=0$ and $D(n)=$ const, when the nonlinear drift term dominates. The respective stationary probability distribution

$$
\begin{gathered}
P(n)=A\left(\frac{D}{\epsilon}+(q-1)(n+1)\right)^{-1 /(q-1)}, \\
A \equiv(2-q)\left(\frac{D}{\epsilon}+(q-1)\right)^{(2-q) /(q-1)},
\end{gathered}
$$

increases monotonically with decreasing $n$, i.e. (with growing the cluster of walker positions) and transforms to the Tsallis distribution at the highest level $n=0$ corresponding to the whole system [3]. Using the generalized definition of entropy, it is easy to show that distribution (10) at $q \neq 1$ corresponds to the nonadditive statistical system for which the $q<1$ and $q>1$ cases are equivalent [9]. As will be seen below, the first case corresponds to the infinitely growing asymptotic of the probability density, and, therefore, it will be assumed that $q>1$.

In the nonstationary case, the analytical description can be carried out in the self-similar regime, when the system behavior is determined by the time dependence $n_{c}(t)$ of the characteristic hierarchy scale, while the probability distribution is represented by a homogeneous function $P(n, t)=n_{c}^{\alpha}(t) \pi(v), v \equiv n / n_{c}$ [10]. If the normalization condition

$$
\int_{0}^{\infty} P(n, t) d n=1,
$$

is satisfied, the leading contribution comes from the drift term caused by the inhomogeneity of the ultrametric space. Then, the exponent $\alpha=-1$, and the self-similar regime is established if the condition $n_{c}^{q-1} \dot{n}_{c}=$ const $\equiv C / \tau_{\mathbf{k}}$ and the equation $\left(\epsilon q \pi^{q-1}-C v\right) \pi^{\prime}-C \pi=0$ are satisfied (hereafter, the prime means the differentiation with respect to the corresponding argument). The solution has the form $\pi^{q-1}=(C / \epsilon) \vee$ and is valid for times $t \ll \tau_{d}$, where $\tau_{d} \equiv\left(\epsilon^{q-2} / D^{q-1}\right) n^{q} \tau_{\mathbf{k}}$. When the drift and the diffusion contributions are of the same order $\left(t \sim \tau_{d}\right)$, the normalization of the distribution over hierarchical levels breaks down [2] and the conditions $\alpha(q-1)+1=0, n_{c} \dot{n}_{c}=C / \tau_{\mathbf{k}}$ and the equation $D \pi "+$ $\left(\epsilon q \pi^{q-1}-C v\right) \pi^{\prime}+\alpha C \pi=0$ must be satisfied to ensure the self-similar regime. The corresponding solution has asymptotics $\pi^{q-1} \longrightarrow(q-1)^{-1}(D / \epsilon) \nu^{-1}$ at $\nu \longrightarrow 0$ and $\pi^{q-1} \longrightarrow(2 C / q \epsilon) \vee$ at $\vee \longrightarrow \infty$. The first asymptotic occurs at large times $t \gg \tau, \tau \equiv\left(n^{2} / C\right) \tau_{\mathbf{k}}$ and the second one, at small times $t \ll \tau$.

Thus, for $F(n)=0$ and $D(n)=$ const, the contribution of the diffusion term at the initial stage $t \ll \tau_{d}$ is negligibly small, so that the distribution over the levels is normalized by the ordinary condition. In this case, the characteristic hierarchy scale increases with time fol- 
lowing the power law $n_{c}^{q}=q C\left(t / \tau_{\mathbf{k}}\right)$ (the lower levels become increasingly substantial), while the probability density decreases hyperbolically, $P^{q-1}(n, \quad t)=$ $(n / q \epsilon)\left(t / \tau_{\mathbf{k}}\right)^{-1}$, the lower the level, the more rapid the decrease (it also follows from this that $q>1$ ). The transition to the diffusion stage proceeds rapidly for higher levels and results in the modification of the time dependence $n_{c}(t)$ to the ordinary square root form $n_{c}=$ $\sqrt{2 C\left(t / \tau_{\mathbf{k}}\right)}$ at $\tau_{d} \sim t \ll \tau$ but the probability density decreases hyperbolically as at the initial stage. With further time increase to macroscopic values $t \gg \tau$, the $n_{c}(t)$ dependence remains unchanged and the probability distribution assumes the asymptotic form $P^{q-1}(n) \longrightarrow(q-1)^{-1}(D / \epsilon) n^{-1}$ corresponding to the stationary distribution (10) at $n \gg D / \epsilon$.

In the presence of an external force $F(n)=$ const and the multiplicative noise $[D(n) \neq$ const], the above-mentioned behavior is realized only at small times $t \ll n(F-$ $\left.D^{\prime}\right)^{-1} \tau_{\mathbf{k}}$. If the opposite condition $n+\left(F-D^{\prime}\right)\left(t / \tau_{\mathbf{k}}\right) \gg$ $\left[\epsilon /\left(F-D^{\prime}\right)\right]^{1 /(q-1)}$ is fulfilled, the characteristic scale increases linearly, $n_{c}=C\left(t / \tau_{\mathbf{k}}\right)$, and the probability decreases as $P(n, t)=\left[n+\left(F-D^{\prime}\right)\left(t / \tau_{\mathbf{k}}\right)\right]^{-1}$. The stationary distribution takes the exponential form $P(n) \propto$ $D^{-1} \exp \left\{\int(F / D) d n\right\}$, which, however, does not mean the presence of additivity. Indeed, since the same dependence $D(n)$ governs the diffusion process both for the whole system and for its parts, the condition of multiplicativity of probabilities breaks down: $P_{1,2}(n)=$ $D(n) P_{1}(n) P_{2}(n)$, where the subscripts indicate the macroscopic components. Therefore, the system with the multiplicative noise is nonadditive even with the Boltzmann's definition of entropy. ${ }^{1}$

\footnotetext{
${ }^{1}$ Note that the term "multiplicative noise" bears no relation to the property of multiplicativity of the corresponding probabilities but reflects the fact that the noise originates from the fluctuation of the kinetic coefficient of the force acting on the system.
}

The above analysis demonstrates that the superdiffusion of a hierarchical system free of external action $(F=0)$ proceeds nonadditively. An assumption of this sort is at the basis of the works of Tsallis et al. [9], where the nonadditivity is postulated in the definition of the transition probabilities $f(\mathbf{x})$ and, moreover, the Fokker-Planck equation is assumed to be nonlinear. With the approach proposed in this paper, both nonlinearity and nonlinearity-induced nonadditivity result from the hierarchical structure. This allows the conclusion to be drawn that free hierarchical systems are invariably nonadditive.

I am grateful to C. Tsallis for kindly placing the results on nonadditive systems at my disposal.

\section{REFERENCES}

1. J.-P. Bouchaud and A. Georges, Phys. Rep. 195, 127 (1990).

2. D. H. Zanette, Braz. J. Phys. 29, 108 (1999); condmat/9905064.

3. A. I. Olemskor̆, Pis'ma Zh. Éksp. Teor. Fiz. 69, 391 (1999) [JETP Lett. 69, 423 (1999)].

4. K. V. Chukbar, Zh. Éksp. Teor. Fiz. 108, 1875 (1995) [JETP 81, 1025 (1995)].

5. R. Rammal, G. Toulouse, and M. A. Virasoro, Rev. Mod. Phys. 58, 765 (1986).

6. A. I. Olemskoi, Fractals in Condensed Matter Physics, in Physics Reviews, Ed. by I. M. Khalatnikov (Gordon and Breach, London, 1996), Vol. 18, Part 1.

7. A. I. Olemskoi and A. D. Kiselev, Phys. Lett. A 247, 221 (1998).

8. E. M. Lifshitz and L. P. Pitaevski1̌, Physical Kinetics (Nauka, Moscow, 1979; Pergamon Press, Oxford, 1981).

9. C. Tsallis, in Nonextensive Statistical Mechanics and its Applications, Lecture Notes in Physics, Ed. by S. Abe and Y. Okamoto (Springer-Verlag, Berlin, 2000).

10. A. I. Olemskoŭ, Usp. Fiz. Nauk 168, 287 (1998) [Phys. Usp. 41, 269 (1998)].

Translated by R. Tyapaev

SPELL: ansatz 